\title{
Solving metallic quantum criticality in a casino
}

\section{Monte Carlo Studies of Quantum Critical Metals}

Authors: E. Berg, S. Lederer, Y. Schattner, and S. Trebst

Annual Reviews of Condensed Matter Physics, arXiv:1804.01988 (2018)

2. Superconductivity mediated by quantum critical antiferromagnetic fluctuations: The rise and fall of hot spots

Authors: X. Wang, Y. Schattner, E. Berg, and R. M. Fernandes

Physical Review B 95, 174520 (2017)

\section{Itinerant Quantum Critical Point with Fermion Pockets and Hot Spots} Authors: Z-H Liu, G. Pan, X-Y Xu, K. Sun, and Z-Y Meng arXiv:1808.08878 (2018)

\section{Recommended with a Commentary by Andrey V Chubukov, University of Minnesota}

One of the most extensively studied items in modern physics of correlated metals is whether a Fermi-liquid (FL) behavior can be destroyed in dimensions $D>1$. Two main roots to non-FL physics have been proposed. One is to increase interactions and bring the system close to a transition to a Mott insulator. Another is to keep interactions relatively weak, but vary some parameter $x$, which can be either doping, or pressure, or a magnetic field, and bring the system to an instability towards a spin or a charge order, either with zero momentum (a Pomeranchuk order), or with a finite momentum (a spin or charge density wave). Near the transition line $T_{c r}(x)$ the dominant interaction between fermions is mediated by fluctuations of a near-critical order parameter. The physics becomes particularly interesting when $x$ is adjusted to reach the quantum-critical point $(\mathrm{QCP}) T_{c r}(x)=0$, because then the dynamics of the pairing interaction becomes relevant, and critical dynamic fluctuations may destroy a DL behavior at $T=0$. Perturbative one-loop calculations at various QCP's in $D \leq 3$ have shown that the fermionic self-energy at $T=0$ indeed has a non-FL form $\Sigma \propto \omega^{1-\alpha}$, with $\alpha<1$, either on the whole FS, or at special FS points - hot spots. There have been numerous efforts to go beyond one-loop and sum up particular series of diagrams. The ultimate desire was to obtain the exact non-FL forms of fermionic and bosonic propagators in $D>1$ dimension larger than one. These studies chiefly focused on the two cases in $D=2$ - the $q=0$ nematic QCP and the $(\pi, \pi)$ spin-density-wave (SDW) QCP. The short summary of these studies is the following $[1,2,3]$ : (i) Higher-loop corrections are logarithmically singular. They generate anomalous dimensions for fermionic and bosonic propagators, and, in the SDW case, also shift the dynamical exponent $z$ from initial $z=2$ towards a smaller value, possibly $z=1$ [1]; 
(ii) A naked QCP is actually inaccessible as it is surrounded by a superconducting dome. Superconductivity is mediated by the same interaction, which gives rise to a non-FL behavior, and competes with a non-FL. There is no obvious small or large parameter at a QCP, and the results (i) and (ii) have been obtained within approximate computation procedures (e.g., by using Eliashberg theory for superconductivity and anomalous dimensions). Because of this limitation, there is a clear need to analyze non-FL physics at a metallic QCP using a different, non-perturbative approach.
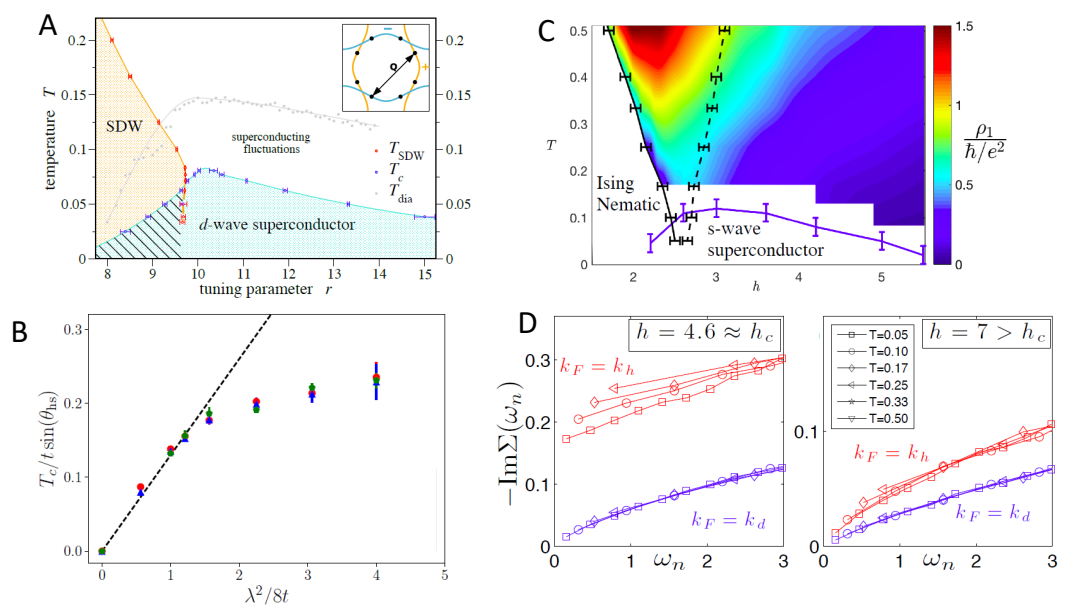

Figure 1: (a-b) A SDW QCP. (a) - Phase diagram with SDW and d-wave superconducting phases, (b) $T_{c}$ vs the coupling $\lambda$. Dashed line is the one-loop result. (c-d)- A nematic QCP. (s) The phase diagram with nematic and s-wave superconducting phases. Color coding is for the exponent for the resistivity;(d) Fermionic self-energy in hot(red) and cold (blue) regions at a QCP $\left(h=h_{c}\right)$ and away from it. Figs. (a,c,d) from Ref. [4], Fig. (b) from Ref. [5].

The papers which I recommend for reading are on this alternative approach - the numerical Quantum Monte Carlo studies of quantum criticality in a metal. I selected three publications - the review by Berg el al [4] (which contains references to earlier works by the extended group), the paper by S. Wang et al [5] on the analysis of superconductivity near a SDW QCP and a detailed comparison with the one-loop Eliashberg calculation of $\mathrm{d}$-wave $T_{c}$, and the very recent paper by Z-H Liu et al [6], also on SDW QCP, in which they argue that they detected the anomalous exponent for the bosonic propagator. There have also been QMC studies of CDW order in the cuprates [7] and of quantum criticality near a topological transition [8]. QMC studies require some care, particularly in case of SDW and CDW transitions, to avoid fermionic sign problem. This is done by using lattice models with at least two bands [9]. Another restriction is the system size both in spatial and in time direction, which does not allow to consider large systems and very low temperatures. There is growing interest to combine QMC and machine learning to focus on fermion modes that are directly associated with low-energy physics [10]. This will potentially allow QMC studies to probe lower temperatures and larger system sizes.

One result, firmly established by QMC, is that both nematic and SDW metallic QCP are surrounded by superconducting domes (see Fig.1, a-b). The authors of Ref. [5] made a 
detailed comparison of their QMC data for SDW QCP with the Eliashberg theory for the hot spot low-energy model and found a near-perfect agreement at small and moderate couplings (Fig. $1 \mathrm{c}$ ), despite that corrections to the Eliashberg theory are of order one even at weak coupling.

Above $T_{c}$, QMC studies of the bosonic propagator at a SDW QCP have found $[4,5,6]$ the dynamical exponent $z=2$, as in one-loop analytical studies. This likely indicates that the scale below which the flow of $z$ becomes visible, is too small for current QMC studies. Nevertheless, in recent QMC work [6], Z-H Liu et al reported that they detected the anomalous dimension of the bosonic propagator $\chi\left(q, \Omega_{m}\right) \propto 1 /\left((q-Q)^{2}+\gamma\left|\Omega_{m}\right|\right)^{1-\eta}$, with $\eta \approx 1 / 8$. The anomalous dimension has not been detected in QMC data away from a QCP, i.e., is the system property at the QCP. A future work in this direction is highly desirable. For a nematic QCP, the situation is more nuanced. The static bosonic propagator obeys Ornstein-Zernike form, as is expected from the one-loop analysis. According to the analytical treatment, the frequency dependence should be in the form of Landau damping $\Omega / q$ (this corresponds to $z=3$ ). QMC data at moderate coupling do show features consistent with $\Omega / q$ dependence, if finite size corrections are included, however for larger couplings the dependence is qualitatively different. Why is that is not clear.

The QMC studies of the fermionic properties along Matsubara axis did find that $\Sigma_{k}\left(\omega_{m}\right)$ shows a FL behavior away from a QCP and a non-FL behavior at a QCP, and that deviations from a FL behavior are the strongest for fermions at hot spots, for a SDW QCP, or, for a nematic QCP, for FS points where the form-factor is the largest (Fig. 1d). This is the expected behavior. However, the non-FL self-energy at a QCP and, e.g., at a hot spot, does not display an expected power-law frequency dependence, $\Sigma\left(\omega_{m}\right) \propto \omega_{m}^{1-\alpha}$. Instead, $\Sigma_{k}\left(\omega_{m}\right)$ becomes weakly dependent on frequency. This well may be due to thermal fluctuations, because $\Sigma_{k}\left(\omega_{m}\right)$ in QMC is obtained at a finite and not that small $T$, but this has not been resolved yet.

So, where do we go from here? My personal view is that at the moment QMC studies confirmed that a phase diagram of a QC metal has a dome of superconductivity around a QCP (i.e., the pairing wins the competition with a non-FL), and that immediately above the dome fermionic self-energy has a non-Fl form and the bosonic propagator shows the anomalous dimension in the SDW case. They also questioned some features of the lowenergy theory, which were taken for granted, like $z=3$ scaling at a nematic QCP. My take is that the QMC results and the results of analytical studies of low-energy models will eventually converge. However, QMC is also capable to explore the regime which was not probed before. Namely, the vast majority of low-energy analytical studies is done under the assumption that the fermion-boson coupling is smaller than the Fermi energy. QCP studies can go outside this regime and study the QC behavior at stronger couplings. This will likely reveal a novel NFL physics. Another item, which QMC studies recently began exploring, is the transport near a QCP. Finally, there are many others QCP's, which so far have not been analyzed using QMC.

And the "big" issue is the relation of both QMC and analytical studies of QC metals to superconductivity, non-FL physics, and other features of the phase diagrams of cuprate, Febased, and even heavy-fermion superconductors. Linear in $T$ resistivity is indeed the central issue here. In many QC theories, resistivity does show a linear behavior, but only above a certain finite $T_{0}$. In analytical studies, this $T_{0}$ is determined only by order of magnitude. 
QMC studies of transport near a metallic QCP can well determine $T_{0}$ with high accuracy and either boost or invalidated the explanations based on metallic quantum criticality. Another issue is thermodynamics, e.g., the $T$ dependence of the specific heat, $C(T)$ in the non-FL regime. According to analytical studies, $C(T) \propto T \log T$ at a SDW QCP and $C(T) \propto T^{2 / 3}$ at a nematic QCP. The $T \log T$ behavior agrees with the observed $C(T)$ in the cuprates, but the true comparison requires one to know the prefactor. This is another area where QCP studies are expected to produce definite results, which one can directly compare with the experiments.

\section{References}

[1] S.-S. Lee, Phys. Rev. B 80, 165102 (2009), M.A. Metlitski and S. Sachdev, Phys. Rev. B 82 075127, (2010); P. Lunts, A. Schlief, and S-S. Lee, Phys. Rev. B 95, 245109 (2017); H. Tobias and W. Metzner, Phys. Rev. B 92, 245128 (2015).

[2] A. Abanov, A. V. Chubukov and J. Schmalian, Adv. Phys. 52, 119 (2003); M.A. Metlitski and S. Sachdev, Phys. Rev. B 82 075128, (2010).

[3] N. E. Bonesteel, I. A. McDonald, and C. Nayak Phys. Rev. Lett. 77, 3009 (1996); C. M. Varma, Phys. Rev. B 55, 14554 (1997); Ar. Abanov, A. V. Chubukov, and A.M. Finkelstein, Europhys. Lett. 54, 488 (2001); M. A Metlitski, D.F. Mross, S. Sachdev, and T. Senthil, Phys Rev B. 91 115111, (2015); S. Raghu, G. Torroba, and H. Wang, Phys. Rev. B 92, 205104 (2015) Y. Wang et al, Phys. Rev. Lett. 117, 157001 (2016).

[4] E. Berg, S. Lederer, Y. Schattner, and S. Trebst, Annual Reviews of Condensed Matter Physics, arXiv:1804.01988 (2018). See also E. Berg, M. A. Metlitski, and S. Sachdev, Science 338, 1606 (2012); Y. Schattner, S. Lederer, S. A. Kivelson, and E. Berg, Phys. Rev. X 6, 031028 (2016); S. Lederer, Y. Schattner, E. Berg, and S. A. Kivelson, PNAS 114, 4905 (2017); Z.-X. Li, F.Wang, H. Yao, and D.-H. Lee, Science Bulletin 61, 925 (2016).

[5] X. Wang, Y. Schattner, E. Berg, and R. M. Fernandes, Physical Review B 95, 174520 (2017).

[6] Z-H Liu, G. Pan, X-Y Xu, K. Sun, and Z-Y Meng, arXiv:1808.08878 (2018).

[7] Z-X Li, Fa Wang, H. Yao, D-H Lee, Phys. Rev. B 95, 214505 (2017).

[8] F. F. Assaad and T. Grover, Phys. Rev. X 6, 041049 (2016); Y.-Y. He, X. Y. Xu, K. Sun, F. F. Assaad, Z. Y. Meng, and Z.-Y. Lu, Phys. Rev. B 97, 081110 (2018)

[9] E. Berg, M. A. Metlitski, and S. Sachdev, Science 338, 1606 (2012).

[10] see, e.g., Yusuke Nomura, Andrew S. Darmawan, Youhei Yamaji, Masatoshi Imada, Phys. Rev. B 96, 205152 (2017); Yi Zhang, Eun-Ah Kim Phys. Rev. Lett. 118, 216401 (2017); X. Y. Xu, Y. Qi, J. Liu, L. Fu, and Z. Y. Meng, Phys. Rev. B 96, 041119 (2017); Z. H. Liu, X. Y. Xu, Y. Qi, K. Sun, and Z. Y. Meng, arXiv:1801.00127, Xiao-Yu Dong, Frank Pollmann, Xue-Feng Zhang, arXiv:1806.00829 and references therein 